01

\title{
Разделение электрического заряда на фронте испарения (конденсации) воды и льда. Заряд сферических капель
}

\author{
(c) А.В. Шавлов, ${ }^{1,2}$ В.А. Джуманджи, ${ }^{1}$ А.А. Яковенко ${ }^{1}$ \\ ${ }^{1}$ Институт криосферы Земли Тюменского НЦ СО РАН, \\ 625000 Тюмень, Россия \\ ${ }^{2}$ Тюменский индустриальный университет, \\ 625000 Тюмень, Россия \\ e-mail: shavlov@ikz.ru
}

(Поступило в Редакцию 17 августа 2017 г.)

Предложен механизм разделения заряда на фронте испарения (конденсации) воды и льда. Низкое значение коэффициента распределения протонов и гидроксид-ионов между паром и конденсированной фазой меньшее, чем коэффициент распределения молекул воды, приводит к тому, что протоны и гидроксид-ионы накапливаются на фазовом фронте при испарении и истощаются при конденсации. Последующая диффузия неравновесных протонов и гидроксид-ионов приводит к межфазному разделению заряда. На разделение заряда оказывает влияние двойной электрический слой, создаваемый ориентационными дефектами на поверхности воды, льда. Рассчитаны зависимости напряженности электрического поля на плоской поверхности воды и льда от скорости фазового превращения. Выполнены оценки электрического заряда капель воды сферической формы при различной напряженности поля и различных радиусах капель.

DOI: 10.21883/JTF.2018.04.45716.2463

\section{Введение}

Интерес исследователей к изучению происхождения электрического заряда капель воды и частиц льда в облаках и атмосферных осадках связан с важностью решения ряда научных проблем. Во-первых, этот интерес вызван традиционным стремлением к углублению понимания природы атмосферного электричества [1-5]. Во-вторых, изучение заряда водяных частиц может способствовать разрешению загадки быстрого роста капель воды до дождевого размера в облаках [6], где механизм электрокоагуляции может играть важную роль [7]. Еще одна новая проблема состоит в том, чтобы выяснить, как взаимодействие электрически заряженных водяных частиц в атмосфере может приводить к их пространственному упорядочению, к более высокой вязкости воздушнокапельной среды по сравнению с вязкостью чистого воздуха, к влиянию на процессы тепломассопереноса в атмосфере [8]. К электрическим взаимодействиям капель субмикронного размера в воздушно-капельной среде может также иметь отношение известная проблема турбулентности ясного неба [9].

Наиболее популярной точкой зрения относительно механизма заряжения капель воды в атмосфере является представление об избирательной адсорбции ионов воздуха на поверхности воды. Этой точки зрения придерживались выдающиеся российские ученые Воейков, Френкель, Седунов [10-12]. Причиной избирательной адсорбции ионов, предположительно, является существование двойного электрического слоя на поверхности воды. Поле данного слоя направлено в сторону пара и затрудняет адсорбцию положительных ионов воздуха, облегчая адсорбцию отрицательных. Донг и Халлетт [13] объясняли процесс заряжения иначе. Они связывали накопление отрицательного заряда водой с тем, что отрицательно заряженные ионы воздуха быстрее диффундируют к поверхности воды и внедряются в нее, чем положительно заряженные. При этом коэффициенты конденсации и тех и других ионов на поверхности воды полагались одинаковыми. Опыты, выполненные в [13], показали, что поверхность воды не всегда заряжается отрицательно относительно пара. Знак заряда зависит от того, какой процесс преобладает на поверхности воды: испарение или конденсация. При испарении знак заряда воды, как правило, отрицательный, а при конденсации положительный. Лед в процессах испарения и конденсации может заряжаться противоположно воде.

В работе [14] была выдвинута гипотеза о том, что ионами, разделяющимися на поверхности раздела конденсированных фаз воды и пара, являются собственные носители заряда воды - протоны, гидроксид-ионы и ориентационные дефекты, а не ионы воздуха, возникающие под действием земной и космической радиации. Причинами разделения заряда считали накопление неравновесных носителей заряда на фазовом фронте и различие коэффициентов диффузии носителей заряда разного знака. Были рассчитаны межфазные электрическое поле и потенциал при различных скоростях испарения и конденсации. Знак разделенного заряда зависел от направления фазового превращения, а абсолютная величина возрастала с увеличением скорости превращения. Работа имела следующие недостатки: а) не были учтены возможности разделения заряда при выполнении условия фазового равновесия конденсированной фазы воды и пара, б) не учитывалось различие коэффициента конденсации для воды и льда, в) не рассчитывались 
нелинейные варианты задачи, которые наиболее вероятно реализуются для льда.

Целью настоящей работы является свободный от недостатков работы [14] численный расчет электрического поля на поверхности воды (льда) при испарении и конденсации при участии собственных носителей заряда - протонов, гидроксид-ионов и ориентационных дефектов. На основании полученных данных будет оценен эффективный электрический заряд капель воды различного радиуса при различных скоростях фазового превращения.

\section{1. Формулировка задачи}

Рассмотрим плоский контакт воды или льда с паровоздушной смесью при нормальном атмосферном давлении и температурах существования воды или льда. В воде и льде носителями заряда являются протоны, гидроксидионы и ориентационные $L$ - и $D$-дефекты. Равновесные концентрации протонов и гидроксид-ионов на много порядков меньше, чем равновесные концентрации ориентационных дефектов. На этом основании процесс переноса носителей заряда в воде или льде можно разделить на два: 1) процесс диффузии ориентационных $L$ - и $D$-дефектов в самосогласованном электрическом поле, на который малочисленные протоны и гидроксидионы не оказывают заметного влияния, 2) процесс диффузии протонов и гидроксид-ионов в отсутствие самосогласованного электрического поля, уничтожаемого ориентационными дефектами, и в присутствии поля ориентационных дефектов в качестве внешнего. Данный прием аналогичен раздельному рассмотрению диффузии основных и неосновных носителей заряда в полупроводниках [15].

Напомним, что ориентационные дефекты появляются и исчезают при поворотах молекул воды в конденсированной фазе. Они появляются парами, один $L$ - и один $D$-дефект, и исчезают (рекомбинируют) тоже парами. $L$-дефект, несущий отрицательный заряд, соответствует такой ориентации двух ближайших молекул воды, при которой на линии, соединяющей атомы кислорода, нет ни одного протона, $D$-дефект (заряд положительный) два протона на линии, бездефектное состояние - один протон на линии.

В паровоздушной смеси носителями заряда являются протоны и гидроксид-ионы. Ориентационные дефекты в данной среде не существуют, так как молекулы воды вращаются с высокой скоростью и не взаимодействуют друг с другом, за исключением процессов соударения. Считаем, что воздух проницаем для пара достаточно хорошо так, что концентрация пара мало зависит от удаленности от фронта испарения или конденсации. Для этого скорость фазового превращения должна быть невысокой. Коэффициенты диффузии протонов и гидроксид-ионов будем считать сравнимыми с коэффициентом самодиффузии молекул воздуха. В паровоздуш- ной смеси протоны и гидроксид-ионы диффундируют в самосогласованном электрическом поле.

Порядок расчетов будет следующим. Сначала сформулируем и решим задачу диффузии ориентационных дефектов в воде и льде, вычислим самосогласованное поле, которое, другими словами, будем называть полем двойного электрического слоя ориентационных дефектов. Затем сформулируем и решим задачу межфазного разделения протонов и гидроксид-ионов в поле двойного электрического слоя, вычислим электрическое поле в паре вблизи поверхности воды или льда. Далее, с помощью этого поля оценим величину эффективного электрического заряда капель воды сферической формы при различных радиусах капель и различных скоростях испарения или конденсации.

\section{1. Задача для ориентационных дефектов}

Пусть вода или лед, в которых ориентационные дефекты являются основными носителями заряда, занимает область полупространства $x>0$. Уравнения диффузии ориентационных дефектов в воде или льде в самосогласованном электрическом поле в стационарном случае и уравнение Пуассона имеют традиционный вид

$$
\begin{gathered}
D_{n} \frac{d^{2} n}{d x^{2}}+\frac{d}{d x}\left[\left(-v+\mu_{n} E_{0}\right)\left(n+n_{0}\right)\right]-\frac{n+p+n p / n_{0}}{\tau_{0}}=0, \\
D_{p} \frac{d^{2} p}{d x^{2}}+\frac{d}{d x}\left[\left(-v-\mu_{p} E_{0}\right)\left(p+n_{0}\right)\right]-\frac{n+p+n p / n_{0}}{\tau_{0}}=0, \\
\frac{d E_{0}}{d x}=-\frac{e}{\varepsilon \varepsilon_{0}}(n-p),
\end{gathered}
$$

где $n$ и $p$ - величины отклонений концентраций от равновесных значений соответственно $L$-дефектов и $D$-дефектов (далее по тексту „концентрации неравновесных $L$ - и $D$-дефектов“ $) ; n_{0}$ - концентрация равновесных $L$ - или $D$-дефектов; $D_{n}, D_{p}$ и $\mu_{n}, \mu_{p}-$ коэффициенты диффузии и подвижности данных дефектов соответственно; $E_{0}-$ напряженность самосогласованного поля; $\tau_{0}-$ время рекомбинации; $\varepsilon-$ диэлектрическая проницаемость; $\varepsilon_{0}$ - электрическая постоянная; $e-$ элементарный заряд; $v$ - скорость движения среды относительно фронта испарения или конденсации или, другими словами, скорость испарения/конденсации (знак скорости положительный для конденсации и отрицательный для испарения, паровоздушная среда занимает область полупространства $x<0)$.

Обсудим граничные условия для ориентационных дефектов сначала при испарении и конденсации воды, а затем при испарении и конденсации льда. Во-первых, вследствие того, что ориентационные $L$ - и $D$-дефекты существуют только в конденсированной фазе, причем в равных количествах, будем считать, что напряженность электрического поля дефектов на фронте испарения или конденсации равна нулю. Вдали от фронта она также стремится к нулю. Во-вторых, на границе вода-пар всегда происходит обмен молекулами воды. Поток молекул 
из пара в воду равен $a V_{T} N_{\text {wap }} / 2$, где $V_{T}-$ средняя скорость теплового движения молекул в направлении воды, $N_{w a p}-$ концентрация молекул воды в паре, $a-$ коэффициент конденсации молекул, множитель 1/2 показывает, что только половина молекул движется в направлении фазы воды, другая половина движется в обратном направлении. Отметим, что коэффициент конденсации имеет значение, много меньшее единицы, $a=0.04$ [16]. Это обстоятельство указывает на то, что только малая часть молекул, ударяющихся о поверхность воды, прилипает к ней. Прилипание, очевидно, происходит только в том случае, когда молекула встраивается в поверхность без образования ориентационного дефекта, т. е. наиболее энергетически выгодно. Обратный поток молекул из воды в пар формируют те молекулы, которые наиболее слабо связаны с водой, т.е. это молекулы, входящие в состав ориентационных дефектов (таких молекул в воде много, примерно каждая третья). Таким образом, на поверхности воды существует поток исчезновения ориентационных $L$ - и $D$-дефектов, в сумме равный потоку молекул пара в воду. В частности, поток $L$-дефектов равен половине этого потока $j_{n \mid 0}=-a V_{T} N_{w a p} / 4$. Отсюда, учитывая общее выражение для потока диффузии в движущейся среде

$$
j_{n \mid 0}=-\left.D_{n} \frac{d n}{d x}\right|_{0}+v\left(\left.n\right|_{0}+n_{0}\right),
$$

получим

$$
\left.\frac{d n}{d x}\right|_{0}=\frac{a V_{T} N_{w a p} / 4+v\left(\left.n\right|_{0}+n_{0}\right)}{D_{n}} \approx \frac{a N_{w a p} V_{T}}{4 D_{n}} .
$$

Отметим, что слагаемое $a V_{T} N_{w a p} / 4$ много больше слагаемого $\left|v\left(\left.n\right|_{0}+n_{0}\right)\right|$ при разумных значениях скорости $v$, например $|v|<10^{-3} \mathrm{~m} / \mathrm{s}$. Для $D$-дефектов получим аналогичное выражение. Таким образом, поверхностный слой воды обеднен ориентационными дефектами. А изза различия коэффициентов диффузии положительно и отрицательно заряженные дефекты оказываются разделенными в пространстве. В результате на поверхности воды существует двойной электрический слой ориентационных дефектов даже в условиях равновесия фаз воды и пара.

Граничные условия для ориентационных дефектов в воде имеют вид

$$
\begin{gathered}
\left.E\right|_{0}=0, \\
\left.\frac{d n}{d x}\right|_{0}=\frac{a N_{w a p} V_{T}}{4 D_{n}}, \\
\left.\frac{d p}{d x}\right|_{0}=\frac{a N_{w a p} V_{T}}{4 D_{p}} .
\end{gathered}
$$

Теперь рассмотрим граничные условия для ориентационных дефектов в твердом агрегатном состоянии воды - во льде. Коэффициент конденсации для льда близок к единице, $a=1$ [17]. Это означает, что все молекулы воды, ударяющиеся в паре о поверхность льда, встраиваются в решетку льда. Если представить молекулу в виде шара, одна половина которого заряжена положительно, а другая отрицательно, то молекула будет ударяться о поверхность льда либо положительно, либо отрицательно заряженной стороной. Если ориентации молекул в паре и молекул на поверхности льда случайны, то можно ожидать, что половина молекул встраивается в поверхность льда без образования ориентационных дефектов, а половина с образованием либо $L$-, либо $D$-дефекта. (На самом деле молекула воды образует связи с четырьмя ближайшими соседями в конденсированной фазе. Поэтому ей больше подходит тетраэдрическая модель, и для такой модели количество актов конденсации молекул без образования дефектов может отличаться от половины.) Остановимся на модели молекулы в виде шара. Поток рождения $L$-дефектов на поверхности льда составляет $j_{n \mid 0}=a V_{T} N_{w a p} / 8$. Такой же поток для $D$-дефектов. При обратном переходе молекул воды изо льда в пар поток уничтожения ориентационных дефектов на поверхности льда мал, поскольку мала равновесная концентрация этих дефектов во льду (концентрация дефектов меньше концентрации молекул воды примерно в $10^{7}$ раз). Следовательно, поверхностный слой льда обогащается ориентационными дефектами при обмене молекулами воды между льдом и паром. Из-за различия коэффициентов диффузии ориентационные дефекты формируют на поверхности льда двойной электрический слой, противоположный по полярности двойному слою в воде. Граничные условия для ориентационных дефектов во льду имеют вид

$$
\begin{gathered}
\left.E\right|_{0}=0, \\
\left.\frac{d n}{d x}\right|_{0}=-\frac{a N_{\text {wap }} V_{T}}{8 D_{n}}, \\
\left.\frac{d p}{d x}\right|_{0}=-\frac{a N_{w a p} V_{T}}{8 D_{p}} .
\end{gathered}
$$

Метод решения. Нелинейную систему уравнений (1) решали численным методом Рунге-Кутта четвертого порядка, реализованным в функции rkfixed(init, x1, x2, intvls, D) программы Mathcad 15.0. На поверхности конденсированной фазы задавали граничные условия (2) или (3) и подбирали значения концентраций $n$ и $p$ таким образом, чтобы вдали от поверхности напряженность электрического поля и концентрации неравновесных дефектов стремились к нулю.

Линейный случай. Рассмотрение линейной задачи интересно потому, что позволит в явном виде получить связь напряженности поля с параметрами дефектов, с интенсивностью обмена фаз молекулами. Для получения линейной задачи воспользуемся системой уравнений (1). Пренебрежем в ней слагаемыми, содержащими произведения $n_{p}, E_{0} n, E_{0} p$, что соответствует приближению малых концентраций неравновесных дефектов, $n, p \ll n_{0}$. $\mathrm{C}$ помощью простейших преобразований исключим из системы уравнений функции $p$ и $E_{0}$ и получим дифференциальное уравнение для концентрации $n$ четвертого 
порядка по производной. Подставим в него пробное решение в виде $n \sim \exp \left(k_{i} x\right)$ и получим характеристическое алгебраическое уравнение четвертой степени по $k_{i}$

$$
\begin{aligned}
k_{i}^{4}-\frac{v}{D} k_{i}^{3} & +\left(\frac{v^{2}}{D_{n} D_{p}}-\frac{2}{L^{2}}-\frac{1}{D \tau_{0}}\right) k_{i}^{2} \\
& +\left(\frac{v}{L^{2} D}+\frac{2 v}{D_{n} D_{p} \tau_{0}}\right) k_{i}+\frac{2}{L^{2} D \tau_{0}}=0
\end{aligned}
$$

где $k_{i}$ - корень уравнения, $D=\frac{D_{n} D_{p}}{D_{n}+D_{p}}, L^{2}=\frac{\varepsilon \varepsilon_{0} k T}{e^{2} n_{0}}$. Физический смысл имеют отрицательные корни. Таких корней два. При малой скорости, например $|v|<10^{-3} \mathrm{~m} / \mathrm{s}$ (малая скорость должна удовлетворять условию $\left.|v| \ll\left(\sqrt{\frac{D}{\tau_{0}}}, \frac{D}{L}\right)_{\min } \approx 1 \mathrm{~m} / \mathrm{s}\right)$, это $k_{1}=-\frac{\sqrt{2}}{L}$, $k_{2}=-\frac{1}{\sqrt{D \tau_{0}}}$. В результате решение для концентрации имеет вид $n=C_{1} \exp \left(k_{1} x\right)+C_{2} \exp \left(k_{2} x\right)$. Функции $p$ и $E_{0}$ выражаются через $n$. Необходимо два граничных условия для определения констант $C_{1}$ и $C_{2}$. Например, для воды это могут быть любые два условия из (2). Для льда также любые два условия из (3).

\section{2. Задача для протонов и гидроксид-ионов}

Движение протонов и гидроксид-ионов в окружении многочисленных ориентационных дефектов в воде и льде можно описать с помощью уравнений диффузии. Напряженность самосогласованного поля $E$ будем считать равной нулю, так как незначительное перемещение ориентационных дефектов мгновенно компенсирует это поле [15]. На перемещение протонов и гидроксид-ионов влияет внешнее поле двойного слоя $E_{0}$. Система уравнений в воде и льде $(x>0)$ имеет следующий вид:

$$
\begin{gathered}
D_{m 2} \frac{d^{2} m}{d x^{2}}+\frac{d}{d x}\left[\left(-v+\mu_{m 2} E_{0}\right)\left(m+m_{2}\right)\right] \\
-\frac{m+q+m q / m_{2}}{\tau_{2}}=0, \\
D_{q 2} \frac{d^{2} q}{d x^{2}}+\frac{d}{d x}\left[\left(-v-\mu_{q 2} E_{0}\right)\left(q+m_{2}\right)\right] \\
-\frac{m+q+m q / m_{2}}{\tau_{2}}=0,
\end{gathered}
$$

где $m, q-$ концентрации неравновесных гидроксидионов и протонов соответственно; $m_{2}-$ концентрация равновесных гидроксид-ионов или протонов; $D_{m 2}, D_{q 2}$ и $\mu_{m 2}, \mu_{q 2}-$ коэффициенты диффузии и подвижности гидроксид-ионов и протонов соответственно; $\tau_{2}$ - время рекомбинации; $v$ - скорость испарения или конденсации ( $v<0$ при испарении, $v>0$ при конденсации).

В паре протоны и гидроксид-ионы движутся в самосогласованном поле $E$. Поле двойного слоя $E_{0}$ отсутствует. Система уравнений в паре $(x<0)$ имеет следующий вид:

$$
\begin{gathered}
D_{m 1} \frac{d^{2} m}{d x^{2}}+\frac{d}{d x}\left[\left(-v_{1}+\mu_{m 1} E\right)\left(m+m_{1}\right)\right] \\
-\frac{m+q+m q / m_{1}}{\tau_{1}}=0, \\
D_{q 1} \frac{d^{2} q}{d x^{2}}+\frac{d}{d x}\left[\left(-v_{1}-\mu_{q 1} E\right)\left(q+m_{1}\right)\right] \\
-\frac{m+q+m q / m_{1}}{\tau_{1}}=0, \\
\frac{d E}{d x}=-\frac{e}{\varepsilon_{1} \varepsilon_{0}}(m-q),
\end{gathered}
$$

где $\varepsilon_{1}$ - диэлектрическая проницаемость пара, $v_{1}$ скорость движения пара относительно фронта испарения или конденсации (знаки $v_{1}$ и $v$ совпадают). Остальные обозначения аналогичны обозначениям в (5), индекс „1““ указывает принадлежность пару.

Составим граничные условия.

1) $\left.m\right|_{-0}=\left.k_{m} m\right|_{+0},\left.\quad q\right|_{-0}=\left.k_{q} q\right|_{+0}$,

2) $\left.j_{m, q}\right|_{-0}=\left.j_{m, q}\right|_{+0}$,

3) $\left.\varepsilon_{1} E\right|_{-0}=\frac{\sigma}{\varepsilon_{0}}=-\frac{e}{\varepsilon_{0}} \int_{0}^{\infty}(m-q) d x$.

Граничное условие 1) в (7) определяет концентрации неравновесных гидроксид-ионов и протонов в паре через таковые в конденсированной фазе с учетом их коэффициентов распределения $k_{m}$ и $k_{q}$. (Напомним, что коэффициент распределения равен отношению концентрации частиц в паре к концентрации в конденсированной фазе.) Коэффициенты распределения $k_{m}$ и $k_{q}$ имеют значения около $10^{-11}$ (см. ниже), что на много порядков меньше коэффициента распределения молекул воды $k_{\mathrm{H}_{2} \mathrm{O}} \approx 10^{-5}$. Это означает, что при переходе гидроксидионов и протонов из конденсированной фазы в пар необходимо преодолеть потенциальный барьер, больший, чем требуется молекуле воды при испарении. В результате оказывается, что при испарении лишь незначительная часть протонов и гидроксид-ионов может преодолеть барьер и перейти из конденсированной фазы в пар. Основная часть носителей, поступающих к фронту испарения вследствие перемещения среды, накапливается у фронта. Различие коэффициентов диффузии носителей приводит к разделению заряда в пространстве и к электрическому заряжению конденсированной фазы относительно пара. В обратном процессе - процессе конденсации водяного пара, носители заряда в конденсированной фазе, наоборот, истощаются, диффузия протекает в обратном направлении, и заряд конденсированной фазы оказывается противоположным тому, который возникает при испарении.

Граничное условие 2) в (7) показывает, что потоки гидроксид-ионов и протонов в паре равны потокам в 
конденсированной фазе, где, например, для гидроксидионов потоки имеют вид

$$
\begin{gathered}
\left.j_{m}\right|_{-0}=-\left.D_{m 1} \frac{d m}{d x}\right|_{-0}+\left(v_{1}-\left.\mu_{m 1} E\right|_{-0}\right)\left(\left.m\right|_{-0}+m_{1}\right), \\
\left.j_{m}\right|_{+0}=-\left.D_{m 2} \frac{d m}{d x}\right|_{+0}+v\left(\left.m\right|_{+0}+m_{2}\right) .
\end{gathered}
$$

Граничное условие 3) в (7) выражает напряженность электрического поля в паре через плотность поверхностного заряда $\sigma$ конденсированной фазы. Отметим, что поверхностный заряд обеспечивают гидроксид-ионы и протоны. Ориентационные дефекты вклада не дают, так как их суммарный заряд равен нулю.

Для решения задачи (5)-(7) использовали численный метод Рунге-Кутта четвертого порядка. Применяли методику стрельбы. Для этого в конденсированной фазе вдали от фронта испарения (конденсации), где задача является заведомо линейной, подбирали значение концентрации $m$ ( $q$ в данной точке рассчитывали по значению $m$ на основе линейного уравнения) таким образом, чтобы после сшивки решений на границе $x=0$ решения для $m, q, E$ в паре вдали от фронта стремились к нулю.

Линейный случай. Линейный случай задачи (5) и (6) реализуется при слабом возмущении равновесных концентраций гидроксид-ионов и протонов. В соответствии с этим пренебрежем в (5) и (6) слагаемыми, содержащими произведения $m q, E m, E q$. Поле двойного слоя $E_{0}$ перераспределяет концентрации гидроксид-ионов и протонов в конденсированной фазе в тонком слое вблизи поверхности. Уберем слагаемое, содержащее $E_{0}$, из системы уравнений (5) и позже учтем влияние этого поля в граничных условиях. Тогда характеристическое уравнение системы (5) в линейном случае будет иметь следующий вид:

$$
k_{i}^{3}-\frac{v}{D_{2}} k_{i}^{2}+\left(\frac{v^{2}}{D_{m 2} D_{q 2}}-\frac{1}{D_{2} \tau_{2}}\right) k_{i}+\frac{2 v}{D_{m 2} D_{q 2} \tau_{2}}=0
$$

Характеристическое уравнение системы (6) в линейном случае имеет вид, подобный уравнению (4) с параметрами $v_{1}, D_{1}, \tau_{1}, D_{m 1}, D_{q 1}, L_{1}$, где

$$
D_{1,2}=\frac{D_{m 1, m 2} D_{q 1, q 2}}{D_{m 1, m 2}+D_{q 1, q 2}}, L_{1}^{2}=\frac{\varepsilon_{1} \varepsilon_{0} k T}{e^{2} m_{1}}, L_{2}^{2}=\frac{\varepsilon \varepsilon_{0} k T}{e^{2} m_{2}} .
$$

В конденсированной фазе $(x>0)$ имеют физический смысл отрицательные корни уравнения (9). Таких корней два при $v>0$ и один при $v<0$. В паре $(x<0)$ должны быть положительные корни. Их два, согласно (4). Таким образом, для решения линейной задачи потребуются три или четыре граничных условия.
Граничные условия следующие:

1) $\left.m\right|_{-0}+m_{1}=k_{m \varphi}\left(\left.m\right|_{+0}+m_{2}\right)$,

2) $\left.q\right|_{-0}+m_{1}=k_{m \varphi}\left(\left.q\right|_{+0}+m_{2}\right)$,

3) $\left.j_{m}\right|_{-0}=\left.j_{m}\right|_{+0}$,

4) $\left.\varepsilon_{1} E\right|_{-0}=\frac{\sigma+\sigma_{\varphi}}{\varepsilon_{0}}=-\frac{e}{\varepsilon_{0}} \int_{0}^{\infty}(m-q) d x+\frac{2 \varepsilon \varphi L}{L_{2}^{2}}$.

Поле двойного электрического слоя $E_{0}$ дает скачок электрического потенциала на границе $\varphi=\int_{0}^{\infty} E_{0} d x$, который изменяет величины коэффициентов распределения гидроксид-ионов и протонов:

$$
k_{m \varphi}=k_{m} \exp \left(-\frac{e \varphi}{k T}\right), k_{q \varphi}=k_{q} \exp \left(-\frac{e \varphi}{k T}\right) .
$$

Первое и второе граничные условия в (10) сшивают концентрации носителей заряда слева и справа от фазового фронта с учетом новых значений коэффициентов распределения. Третье граничное условие выражает равенство потоков носителей слева и справа от фронта. Четвертое граничное условие определяет величину напряженности электрического поля в паре через плотность поверхностного заряда воды $\sigma$ и дополнительную плотность заряда $\sigma_{\varphi}$, создаваемую скачком потенциала в двойном электрическом слое:

$$
\begin{aligned}
\sigma_{\varphi} & =e \int_{0}^{\infty}\left(m_{\varphi}-q_{\varphi}\right) d x= \\
& =e m_{2} \int_{0}^{\infty}\left(\exp \left(\frac{e \varphi}{k T}\right)-\exp \left(\frac{e \varphi}{k T}\right)\right) d x \approx \frac{2 \varepsilon \varepsilon_{0} \varphi L}{L_{2}^{2}} .
\end{aligned}
$$

\section{2. Результаты и их обсуждение}

\section{1. Двойной электрический слой ориентационных дефектов}

Параметры воды при температуре $t=20^{\circ} \mathrm{C}$. $n_{0}=6 \cdot 10^{27} \mathrm{~m}^{-3}$ (значение концентрации выбрано таким образом, чтобы величина произведения $n_{0} \tau_{0}$ совпадала с таковой во льде, где $\left(n_{0} \tau_{0}\right)^{-1}$ представляет собой константу скорости рекомбинации дефектов), $D_{n}=4 \cdot 10^{-10} \mathrm{~m}^{2} / \mathrm{s}, D_{p}=2 \cdot 10^{-10} \mathrm{~m}^{2} / \mathrm{s} \quad$ (коэффициенты диффузии выбраны такими же, как во льде, согласно данным [18]). Отметим, что в работе [14] использовали намного меньшие значения коэффициентов диффузии, полагая, что заряженный слой воды имеет большую толщину и электропроводимость осуществляется путем чередования движения ориентационных дефектов и протонов так, что вклад в электропроводимость и тех и других одинаков. В результате 


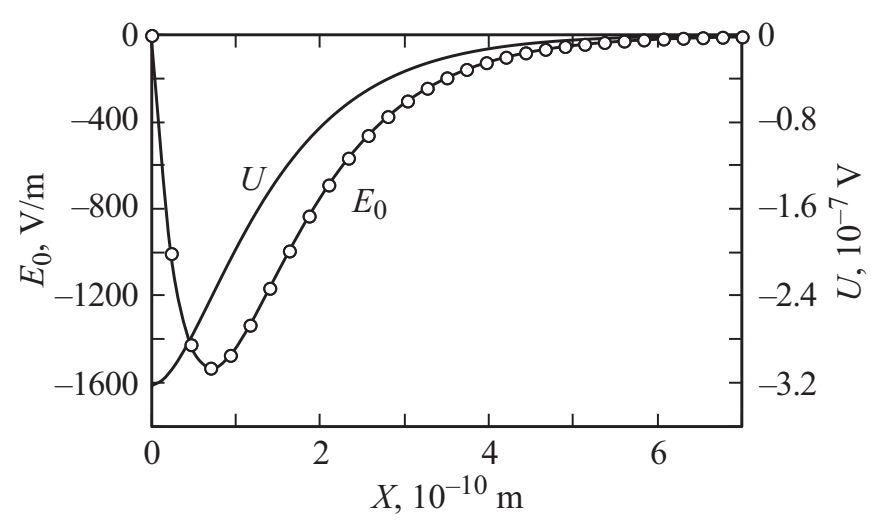

Pис. 1. Напряженность электрического поля $E_{0}$ и падение потенциала $U$ в двойном электрическом слое ориентационных дефектов в воде при температуре $t=20^{\circ} \mathrm{C}$.

диффузия ориентационных дефектов оказывалась стесненной. В настоящей работе уточнено, что реальная толщина двойного электрического слоя в воде равна толщине одного-двух мономолекулярных слоев, вследствие чего указанный в [14] механизм чередования носителей заряда при электропроводимости не реализуется. Далее, $\varepsilon=90[18], \tau_{0}=2 \cdot 10^{-11} \mathrm{~s}-$ время диэлектрической релаксации воды [18], $a=0.04$ [16], $N_{\text {wap }}=P_{\text {wap }} / k T=6 \cdot 10^{23} \mathrm{~m}^{-3}[19], V_{T}=300 \mathrm{~m} / \mathrm{s}$.

Параметры воды при $t=100^{\circ} \mathrm{C}: n_{0}=1.2 \cdot 10^{28} \mathrm{~m}^{-3}$, $D_{n}=4 \cdot 10^{-10} \mathrm{~m}^{2} / \mathrm{sc}, \quad D_{p}=2 \cdot 10^{-10} \mathrm{~m}^{2} / \mathrm{s}, \quad \varepsilon=90$, $\tau_{0}=10^{-11} \mathrm{~s}[18], a=0.04, N_{\text {wap }}=2 \cdot 10^{25} \mathrm{~m}^{-3}$.

Параметры льда при $t=-10^{\circ} \mathrm{C}: \quad n_{0}=$ $=6 \cdot 10^{21} \mathrm{~m}^{-3}[18], D_{n}=4 \cdot 10^{-10} \mathrm{~m}^{2} / \mathrm{s}, D_{p}=2 \cdot 10^{-10} \mathrm{~m}^{2} / \mathrm{s}$, $\varepsilon=100, \tau_{0}=2 \cdot 10^{-5} \mathrm{~s}-$ время диэлектрической релаксации льда [18], $a=1$ [17], $N_{\text {wap }}=1.5 \cdot 10^{23} \mathrm{~m}^{-3}$.

На рис. 1 изображены напряженность электрического поля $E_{0}$ и падение потенциала $U$ в двойном электрическом слое ориентационных дефектов в воде при температуре $t=20^{\circ} \mathrm{C}$, найденные при решении нелинейной задачи. Напряженность достигает $-10^{3} \mathrm{~V} / \mathrm{m}$, а потенциал $-10^{-7}$ В. При не слишком высокой скорости фронта испарения или конденсации, например $|v|<10^{-3} \mathrm{~m} / \mathrm{s}$, напряженность поля и потенциал не зависят от скорости. Обе величины имеют отрицательный знак. Отрицательный знак объясняется тем, что поверхность воды обеднена ориентационными дефектами из-за обмена молекулами воды с паром, и отрицательно заряженные $L$-дефекты быстрее диффундируют к поверхности из глубины воды, чем положительные $D$-дефекты, и поверхностный слой заряжается отрицательно, а более глубокий слой воды - положительно. Концентрации неравновесных ориентационных дефектов по результатам расчетов оказались намного меньше концентрации равновесных, $n, p \ll n_{0}$. В связи с этим фактом можно ожидать, что решения нелинейной задачи совпадут с решениями линейной задачи. На рис. 1 круглыми маркерами показаны значения напряженности поля, вычисленные по аналитической формуле, полученной из линейной задачи (4), (2):

$$
\begin{aligned}
& E_{0}=\frac{e}{\varepsilon \varepsilon_{0}} \frac{a N_{\text {wap }} V_{T} \tau_{0}}{4} \frac{D_{p}-D_{n}}{D_{p}+D_{n}} \\
& \times\left[\left(1-\frac{2 D \tau_{0}}{L^{2}}\right)^{-1}\left(\exp \left(-\frac{\sqrt{2}}{L} x\right)-\exp \left(-\frac{1}{\sqrt{D \tau_{0}}} x\right)\right)\right] .
\end{aligned}
$$

Видим, что значения поля в линейном случае совпадают со значениями в нелинейном.

Проанализируем аналитическую формулу (11). Напряженность поля двойного слоя прямопропорциональна коэффициенту конденсации $a$, концентрации молекул воды в паре $N_{\text {wap }}$, разности коэффициентов диффузии $\left(D_{p}-D_{n}\right)$. Знак напряженности определяется знаком разности коэффициентов диффузии. Сомножитель в квадратных скобках формулы (11) положителен, его величина в двойном слое меньше или порядка единицы. С увеличением температуры концентрация $N_{\text {wap }}$ быстро увеличивается соответственно напряженность поля тоже увеличивается. Так, например, при температуре $t=100^{\circ} \mathrm{C}$ напряженность поля в двойном слое достигает значения $-10^{5} \mathrm{~V} / \mathrm{m}$, а потенциал $-10^{-5} \mathrm{~V}$. Решения нелинейной и линейной задач также совпадают при $t=100^{\circ} \mathrm{C}$.

При испарении и конденсации льда при $t=-10^{\circ} \mathrm{C}$ поверхность льда обогащена ориентационными дефектами из-за обмена молекулами воды с паром. Отрицательно заряженные $L$-дефекты быстрее диффундируют от поверхности вглубь льда, чем положительные $D$-дефекты, и поверхностный слой заряжается положительно, а более глубокий слой льда - отрицательно. В результате электрическое поле и потенциал двойного слоя имеют положительный знак. Поле достигает значения $10^{6} \mathrm{~V} / \mathrm{m}$, а потенциал $10^{-1} \mathrm{~V}$. Задача для льда является существенно нелинейной, $n, p \gg n_{0}$, и аналитическое решение, подобное (11), оказывается непригодным.

\section{2. Межфазное разделение протонов и гидроксид-ионов}

Параметры воды при $t=20^{\circ} \mathrm{C}: m_{2}=6 \cdot 10^{19} \mathrm{~m}^{-3}$, $D_{m 2}=7 \cdot 10^{-9} \mathrm{~m}^{2} / \mathrm{s}, \quad D_{q 2}=10^{-8} \mathrm{~m}^{2} / \mathrm{s} \quad[18], \quad \varepsilon=90$, $\tau_{2}=\left(m_{2} \gamma\right)^{-1}=7 \cdot 10^{-5} \mathrm{~s}$, где $\gamma=2.3 \cdot 10^{-16} \mathrm{~m}^{3} / \mathrm{s}$ константа скорости рекомбинации протонов и гидроксид-ионов $[18], \quad k_{\mathrm{H} 2 \mathrm{O}}=N_{\text {wap }} / N_{\mathrm{H} 2 \mathrm{O}}=2 \cdot 10^{-5}$, где $N_{\mathrm{H} 2 \mathrm{O}}=3 \cdot 10^{28} \mathrm{~m}^{-3}-$ концентрация молекул в воде, $k_{m}=k_{q}=10^{-11}$. Значение коэффициентов $k_{m}, k_{q}$ было рассчитано на основании опытных данных по электропроводности воды, $\sim 6 \cdot 10^{-6} \Omega^{-1} \mathrm{~m}^{-1}$ [18], и электропроводности воздуха у поверхности земли, $10^{-14}-10^{-13} \Omega^{-1} \mathrm{~m}^{-1}[20]$. Внутри облаков электропроводность может быть в десятки раз выше из-за обмена водяных капель и воздуха протонами и гидроксидионами. Поэтому значения коэффициентов $k_{m}, k_{q}$ можно выбирать в десятки раз более высокими. Но в нашей задаче это не имеет существенного значения, так, 


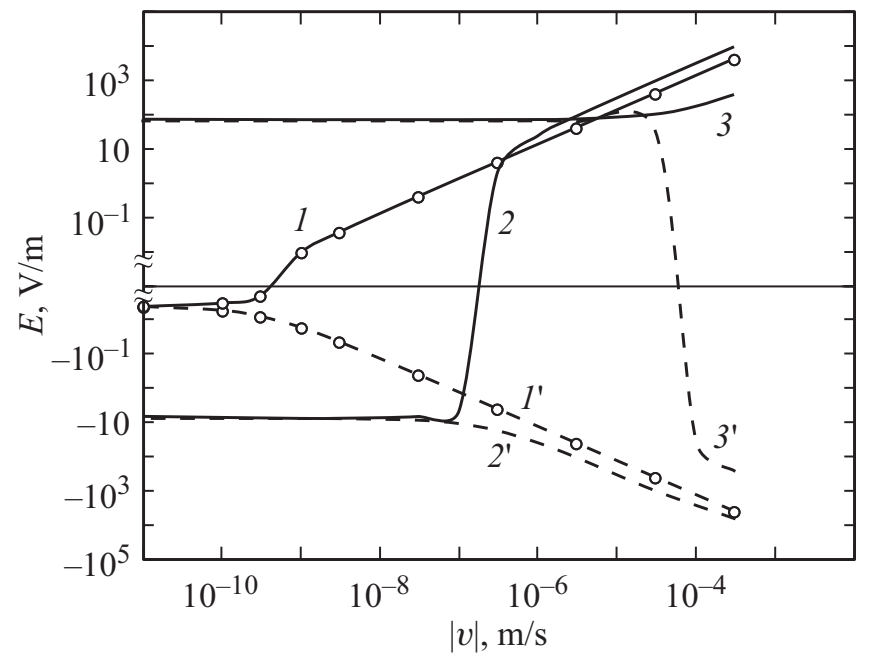

Рис. 2. Зависимость напряженности электрического поля $E$ на поверхности конденсированной фазы от линейной скорости $v$ испарения (конденсации). 1-3 - при испарении воды при температуре $20^{\circ} \mathrm{C}$, воды при $100^{\circ} \mathrm{C}$, льда при $-10^{\circ} \mathrm{C}$ соответственно; $1^{\prime}-3^{\prime}-$ то же при конденсации соответственно.

коэффициенты $k_{m}, k_{q}$, будучи малыми $(\ll 1)$, слабо влияют на искомый результат. Напомним, что мы ищем величину напряженности электрического поля на поверхности воды и льда при испарении (конденсации).

Параметры воды при $t=100^{\circ} \mathrm{C}: m_{2}=10^{22} \mathrm{~m}^{-3}$, $D_{m 2}=7 \cdot 10^{-9} \mathrm{~m}^{2} / \mathrm{s}, \quad D_{q 2}=10^{-8} \mathrm{~m}^{2} / \mathrm{s}, \quad \varepsilon=50, \quad \tau_{2}=$ $=\left(m_{2} \gamma\right)^{-1}=10^{-6} \mathrm{~s}$, где $\gamma \approx 10^{-16} \mathrm{~m}^{3} / \mathrm{s} \quad[18], \quad k_{\mathrm{H} 2 \mathrm{O}}=$ $=6.7 \cdot 10^{-4}, k_{m}=k_{q}=10^{-11}$.

Параметры льда при $t=-10^{\circ} \mathrm{C}: m_{2}=10^{17} \mathrm{~m}^{-3}$, $D_{m 2}=2 \cdot 10^{-8} \mathrm{~m}^{2} / \mathrm{s}, \quad D_{q 2}=2 \cdot 10^{-7} \mathrm{~m}^{2} / \mathrm{s} \quad[18], \quad \varepsilon=100$, $\tau_{2}=\left(m_{2} \gamma\right)^{-1}=7 \cdot 10^{-4} \mathrm{~s}$, где $\gamma=1.5 \cdot 10^{-14} \mathrm{~m}^{3} / \mathrm{s}$ - константа скорости рекомбинации протонов и гидроксидионов $[18], \quad k_{\mathrm{H} 2 \mathrm{O}}=N_{\text {wap }} / N_{\mathrm{H} 2 \mathrm{O}}=5 \cdot 10^{-6}, \quad k_{m}=k_{q}=$ $=10^{-7}$ (электропроводность льда $\sim 10^{-8} \Omega^{-1} \mathrm{~m}^{-1}[18]$ ).

Параметры водяного пара: $m_{1}=m_{2} k_{m}$, $D_{m 1}=10^{-5} \mathrm{~m}^{2} / \mathrm{s}, \quad D_{q 1}=2 \cdot 10^{-5} \mathrm{~m}^{2} / \mathrm{s} \quad$ (считаем, что протон в паровой фазе ассоциирован с молекулой воды и имеет такой же коэффициент диффузии, как молекула $\mathrm{H}_{2} \mathrm{O}, 2 \cdot 10^{-5} \mathrm{~m}^{2} / \mathrm{s}$ [21]; гидроксид-ион тоже ассоциирован с молекулой воды и имеет вдвое меньший коэффициент диффузии, так как более тяжелый). Далее $\tau_{1}=\tau_{2} / k_{m}, \varepsilon_{1}=1, v_{1}=v / k_{\mathrm{H} 2 \mathrm{O}}$.

На рис. 2 представлены зависимости напряженности электрического поля на поверхности воды и льда от линейной скорости испарения (конденсации), найденные при решении нелинейной задачи (5)-(7). При низких скоростях испарения (конденсации) поле не зависит ни от абсолютной величины скорости фазового превращения, ни от знака скорости $(v<0-$ испарение, $v>0$ - конденсация). Оно определяется зарядом конденсированной фазы, приобретенным за счет переноса протонов и гидроксид-ионов в поле двойного слоя. Вода при этом приобретает положительный заряд $(E<0)$, а лед отрицательный заряд $(E>0)$. При высоких скоростях испарения (конденсации) начинает преобладать механизм межфазного разделения заряда, не связанный с воздействием поля двойного слоя. В этом случае электрическое поле $E$ на поверхности конденсированной фазы изменяется пропорционально скорости фазового превращения $v$, взятой с отрицательным знаком $(E \propto-v)$. Механизм разделения заряда здесь заключается в том, что при испарении воды или льда протоны и гидроксидионы накапливаются в избыточном количестве у фронта в конденсированной фазе вследствие существования потенциального барьера для их перехода в пар. Некоторое количество избыточных носителей все же преодолевает барьер и попадает в пар. В большем количестве в пар попадают самые подвижные носители конденсированной фазы - протоны. Они заряжают пар положительно, а конденсированная фаза приобретает отрицательный заряд. При обратном фазовом переходе, при конденсации количество поставляемых из пара в конденсированную фазу гидроксид-ионов и протонов очень мало, и концентрации носителей в конденсированной фазе истощаются у фронта. Далее конденсированная фаза „высасывает носители заряда из пара за счет диффузии. Наиболее быстро в нее поступают самые подвижные носители протоны. Вследствие этого конденсированная фаза заряжается положительно относительно пара.

Расчеты показали, что возмущения концентраций протонов и гидроксид-ионов в области фазового фронта велики для системы пар-лед и малы для системы пар-вода. Можно ожидать, что в случае испарения (конденсации) воды решения нелинейной задачи будут совпадать с решением линейной. Действительно, изображенные круглыми маркерами на рис. 2 значения напряженности поля, рассчитанные в линейной задаче (9), (10), демонстрируют совпадение с решением нелинейной задачи, кривые $1,1^{\prime}$ на рис. 2. Значения напряженности в линейном случае рассчитывали по полученной из (9), (10) аналитической формуле

$$
\left.E\right|_{-0}=-\frac{e m_{2} v \tau_{2}}{\varepsilon_{1} \varepsilon_{0}}\left(\frac{D_{q 2}-D_{m 2}}{D_{q 2}+D_{m 2}}\right)+\frac{2 \varepsilon \varphi L}{\varepsilon_{1} L_{2}^{2}} .
$$

Из формулы (12) следует, что напряженность поля на поверхности воды определяется скоростью $v$ испарения (конденсации), константой скорости рекомбинации протонов и гидроксид-ионов $\gamma=\left(m_{2} \cdot \tau_{2}\right)^{-1}$, величиной и знаком разности их коэффициентов диффузии, $\left(D_{q 2}-D_{m 2}\right)$, а также при низких значениях $v$ величиной поверхностного заряда воды, наведенного двойным электрическим слоем ориентационных дефектов.

\section{3. Заряд водяных капель сферической формы}

Оценим электрический заряд капель воды сферической формы по найденной величине напряженности 


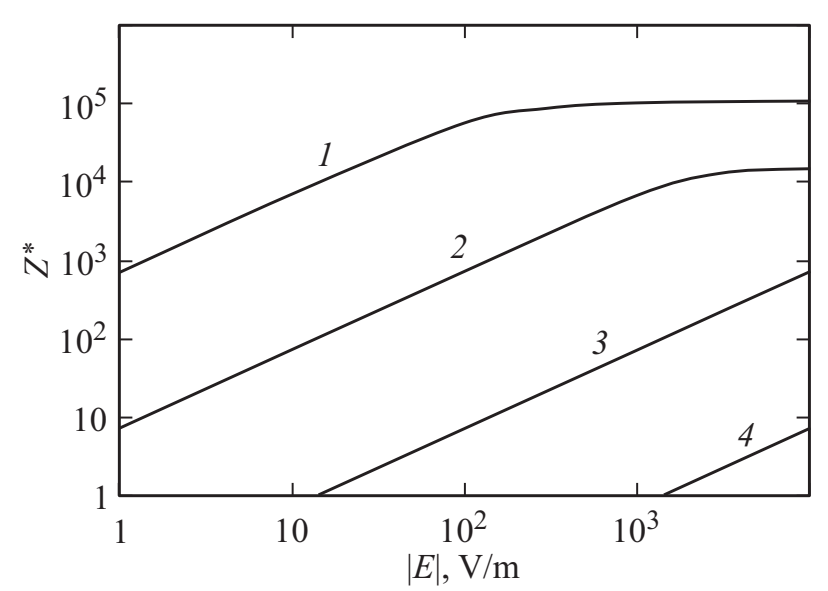

Pис. 3. Эффективный заряд $Z^{*}$ капли воды в зависимости от напряженности электрического поля $E$ на ее поверхности. $1-$ радиус капли $R=10^{-3}, 2-10^{-4}, 3-10^{-5}, 4-10^{-6} \mathrm{~m}$. Радиус Дебая плазмы равен $10^{-3} \mathrm{~m}$.

электрического поля на поверхности воды, используя классическую формулу

$$
E=\frac{e Z}{4 \pi \varepsilon_{1} \varepsilon_{0} R^{2}},
$$

где $Z$ - заряд капли в единицах элементарного заряда, $R$ - радиус капли, $E$ - напряженность поля на поверхности капли. При этом необходимо сделать некоторые замечания. В работе [22] было показано, что для заряженной капли, помещенной в плазму, формулой (13) можно пользоваться в том случае, когда заряд капли мал так, что потенциальная энергия взаимодействия носителя заряда плазмы с каплей еф во много раз меньше кинетической энергии носителя $k T,|e \phi| \ll k T$, где $\phi-$ потенциал электрического поля капли. При большом заряде капли, когда $|e \phi| \gg k T$, вокруг капли формируется прочная плазменная оболочка, имеющая некоторую толщину и заряд, частично компенсирующий заряд капли. На внешние электрические воздействия капля откликается как единое целое с плазменной оболочкой. Таким образом, капля с оболочкой представляет собой частицу с эффективным зарядом $Z^{*}, Z^{*}<Z$ и эффективным радиусом $R^{*}, R^{*}>R$. Эффективный радиус $R^{*}$ определяется из условия $\left|e \phi^{*}\right|=k T$, где $\phi^{*}$ - значение потенциала на поверхности радиуса $R^{*}$. Далее, в (13) под зарядом и радиусом капли будем подразумевать их эффективные значения $Z^{*}$ и $R^{*}$, а под напряженностью поля - напряженность на сферической поверхности радиуса $R^{*}$.

На рис. 3 представлены зависимости эффективного заряда $Z^{*}$ капли от напряженности электрического поля $E$ на ее истинной поверхности при различных истинных значениях радиуса $R$ капли. Заряд может достигать значительной величины, $10^{5}$ элементарных зарядов, при радиусе $10^{-3} \mathrm{~m}$. При низкой напряженности поля эффективный заряд капли совпадает с истинным зарядом и возрастает прямо пропорционально величине напряженности. При большой напряженности поля, когда вокруг капли образуется плазменная оболочка, эффективный заряд насыщается. С увеличением радиуса капли эффективный заряд также возрастает. Значение радиуса Дебая плазмы оказывает слабое влияние на величину эффективного заряда капли.

Для расчета эффективного заряда капли использовали численные решения уравнения Пуассона-Больцмана в сферических координатах

$$
\Delta \phi=\frac{e m_{1}}{\varepsilon_{1} \varepsilon_{0}}\left(\exp \left(\frac{e \phi}{k T}\right)-\exp \left(\frac{-e \phi}{k T}\right)\right),
$$

с граничными условиями

$$
\left.\frac{\partial \phi}{\partial r}\right|_{R}=E,\left.\quad \frac{\partial \phi}{\partial r}\right|_{\infty}=0,
$$

где $E$ - ранее найденное значение напряженности поля на поверхности воды.

\section{Заключение}

Настоящая работа явилась существенным развитием работы [14]. В ней сформулирована и решена нелинейная задача разделения электрического заряда на фронте испарения (конденсации) воды и льда. Детально изучен механизм переноса протонов и гидроксид-ионов через межфазную границу. Особенностью поведения данных носителей является их накопление на фазовом фронте при испарении воды и льда и истощение концентрации носителей при конденсации пара. Изучено образование двойного электрического слоя ориентационных дефектов в конденсированной фазе при ее обмене молекулами воды с паром и оценено влияние двойного слоя на межфазный перенос протонов и гидроксид-ионов. Вычислено электрическое поле на поверхности воды и льда и оценен эффективный электрический заряд капли воды сферической формы с учетом образования плазменной оболочки вокруг капли. Заряд капли воды радиусом $10 \mu \mathrm{m}$ может достигать $10^{3}$ элементарных зарядов, а при радиусе $1 \mathrm{~mm}-10^{5}$. Такие значения согласуются с опытными данными [23]. Таким образом, в работе показано, что для объяснения существования электрического заряда капель облаков и капель атмосферных осадков нет необходимости привлекать участие сторонних ионов воздуха. Достаточно участия собственных ионов воды, протонов и гидроксид-ионов, возникающих при диссоциации молекул воды. Эти собственные ионы заряжают капли в процессах испарения и конденсации.

Для дальнейшего развития и уточнения модели разделения зарядов, рассмотренной в настоящей работе, необходимо накопление подробных экспериментальных данных об электрических свойствах капель воды и частиц льда при различных скоростях процессов испарения и 
конденсации. Таких данных в литературе содержится крайне мало.

В заключение выражаем искреннюю благодарность академику РАН В.П. Мельникову за внимание и всестороннюю поддержку настоящей работы. Также благодарим С.Н. Романюк за многочисленные полезные обсуждения.

\section{Список литературы}

[1] Williams E., Markson R. Heckman S. // Geophys. Res. Lett. 2005. Vol. 32. L19810.

[2] Shimogawa M. Holzworth R.H. // Ann. Geophys. 2009. Vol. 27. P. 1423.

[3] Harrison R.G., Aplin K.L., Rycroft M.J. // J. Atmospheric and Solar-Terrestrial Physics. 2010. Vol. 72. N 5. P. 376.

[4] Кузнецов В.В. Атмосферное электрическое поле: факты, наблюдения, корреляции, модели. М.: Наука, 2004.

[5] Дворников В.М., Балыбина А.С. // Солнечно-земная физика. 2009. № 13. С. 75.

[6] Elperin T., Kleeorin N., Liberman M., Rogachevskii I. // Phys. Fluids. 2013. Vol. 25. P. 085104.

[7] Shavlov A.V., Dzhumandzhi V.A. // J. Aerosol Science. 2016. Vol. 91. P. 54.

[8] Shavlov A.V., Sokolov I.V., Hazan V.L., Romanyuk S.N. // Phys. Scr. 2014. Vol. 89. P. 125402.

[9] Шавлов А.В., Соколов И.В., Джумманджи В.А. // ДАН. 2016. T. 470. № 1. C. 32.

[10] Воейков А.И. Метеорология: в 4-х ч. СПб.: Картографическое заведение А. Ильина, 1904.

[11] Френкель Я.И. Теория явлений атмосферного электричества. М.: Гостехиздат, 1949.

[12] Седунов Ю.С. Физика образования жидкокапельной фазы в атмосфере. Л.: Гидрометеоиздат, 1972.

[13] Dong Y., Hallett J. // J. Geophys. Res. D. 1992. Vol. 97. P. 20361.

[14] Шавлов А.В. // Коллоидный журн. 2009. Т. 71. № 2. С. 267.

[15] Бонч-Бруевич В.Л., Калашников С.Г. Физика полупроводников. М.: Мир, 1977.

[16] Амелин А.Г. Теоретические основы образования тумана при конденсации пара. М.: Химия, 1966.

[17] Бутковский А.В. // ТВТ. 1994. Т. 32. Вып. 5. С. 793.

[18] Эйзенберг Д., Кауиман В. Структура и свойства воды. Л.: Гидрометеоиздат, 1975.

[19] Волков А.И., Жарский И.М. Большой химический справочник. Минск: Современная школа, 2005.

[20] Имянитов И.М., Чубарина Е.В. Электричество свободной атмосферы. Л.: Гидрометеоиздат, 1965.

[21] Кошкин Н.Н., Ширкевич М.Г. Справочник по элементарной физике. М.: Наука, 1976.

[22] Shavlov A.V., Romanyuk S.N., Dzhumandzhi V.A. // Phys. Plasm. 2013. Vol. 20. N 2. P. 023703.

[23] Бабичев А.П., Бабушкина Н.А., Братковский А.М. и др. Физические величины: Справочник / Под ред. И.С. Григорьева, Е.З. Мейлихова. М.: Энергоатомиздат, 1991. 